\title{
WHAT KIND OF NECESSARY BEING COULD GOD BE?
}

\section{RICHARD SWINBURNE}

University of Oxford

\begin{abstract}
A logically impossible sentence is one which entails a contradiction, a logically necessary sentence is one whose negation entails a contradiction, and a logically possible sentence is one which does not entail a contradiction. Metaphysically impossible, necessary and possible sentences are ones which become logically impossible, necessary, or possible by substituting what I call informative rigid designators for uninformative ones. It does seem very strongly that a negative existential sentence cannot entail a contradiction, and so 'there is a God' cannot be a metaphysically necessary truth. If it were such a truth, innumerable other sentences which seem paradigm examples of logically possible sentences, such as 'no one knows everything' would turn out to be logically impossible. The only way in which God could be a logically necessary being is if there were eternal necessary propositions independent of human language or God's will, such that the proposition that there is no God would entail - via propositions inaccessible to us - a contradiction. But if there were such propositions, God would have less control over the universe than he would have otherwise.
\end{abstract}

I.

I shall understand by 'metaphysical necessity' the strongest kind of necessity there is, and by 'metaphysical impossibility' the strongest kind of impossibility there is, and so by 'metaphysical possibility' the weakest kind of possibility there is. My concern in this paper is with whether it is metaphysically possible that God be a metaphysically necessary being in these senses. 
A substance or event is metaphysically necessary (or whatever) iff it is metaphysically necessary (or whatever) that it exists; and since we can have no discussable knowledge of whether it is metaphysically necessary (or whatever) that the substance or event exists, except (at least in part) by reflecting on features of the sentence which asserts this, it will be more convenient to speak of necessity etc. as belonging to the sentence. I shall come back later to the issue of whether these modal properties belong primarily to entities of some other kind, such as propositions, and consider the consequences which would follow if they did.

I begin with general considerations about what determines the meanings, in the sense of the truth conditions, of the sentences of a human language, that is the conditions in which they are true and the conditions in which they are false and so which other sentences they entail and are entailed by. Sentences of a language mean what its speakers (or - in the case of technical terms - some group of experts, e.g. physicists) mean by them. Each of us learns the meanings of certain sentences by being shown many observable conditions under which those sentences are regarded as true or as false, and by being told of other sentences to which a speaker is regarded as committed by uttering those sentences, and other sentences which are such that someone who utters them is regarded as committed to the former sentences. We learn the meaning of a word by being taught the difference to the meaning of a sentence made by that word playing a certain role in the sentence. By being taught the meanings of individual words and of sentences of various forms, we may then come to an understanding of the meaning of a sentence in which those words are arranged in a certain way, even if we have not been shown observable conditions under which that sentence is regarded as true or as false. Showing 'observable conditions' may involve pointing to them or describing them by terms already introduced. For example, we learn the meaning of 'there is a cat over there' by being shown observable circumstances under which this sentence is regarded as true, and observable circumstances under which it is regarded as false; and by being told that someone who utters this sentence is regarded as committed to 'there is an animal over there', and someone who utters 'there are two cats over there' is regarded as committed to the original sentence. We learn the meaning of 'there is a dog over there' in a similar way. Thereby we come to know the meanings of 'cat' and 'dog', and so the kind of meaning possessed by sentences of the form 'there is a $\Phi$ over there.' We need to observe many different examples of 
observable conditions under which a sentence containing a certain word in various roles is regarded as true or false, and of the commitments speakers who use sentences containing that word in various roles are regarded as having; and this allows us to acquire an understanding of the conditions under which some new sentence containing that word would be regarded as true or false. Examples of different observable conditions under which some sentence is true or false, and of sentences to which we are not committed by a given sentence also illustrate which conditions do not rule out the sentence being true. We extrapolate, that is, from a stock of supposedly paradigm examples (of observable conditions and relations of commitment) to an understanding that the sentence would be regarded as true (or false, as the case may be) under conditions sufficiently similar in certain respects to most of the paradigm examples. ${ }^{1}$

Because humans have very similar psychologies determining how they learn meanings, and because members of a language group are exposed to very similar paradigm examples (of observable conditions and rules of commitment), members of the same language group normally acquire a very similar understanding of the meanings of words and sentences. This common understanding may be reinforced by dictionary compilers and philosophers who 'tidy up' language by laying down rules for correct usage, usually by codifying most people's actual usage. The rules give general descriptions of the observable conditions under which various sentences of the language are true and of the observable conditions under which various sentences of the language are false, and of the kinds of other sentences to which a sentence of a given kind commits the speaker and by which sentences of other kinds a speaker is committed to a sentence of a given kind. The rules of the syllogism for example are rules of this latter kind; 'all A's are B' and 'All B's are C' commits one to 'All A's are C'. But such rules can in the end only be understood by examples of observable 'conditions' and 'kinds' of sentences. One couldn't understand the stated rule of the syllogism without being shown some things which have some property, and some things which have another property, and examples of things which constitute 'all' members of a class. This programme of

${ }^{1}$ Note however that the sense of a word which we get from this process may be such as to rule out a few of the supposedly paradigm examples as examples of things to which that word applies. Thus we may derive from many supposedly paradigm examples by which we are taught the meaning of the word 'cat' a sense of 'cat' which rules out one of these examples as being a cat at all; it might turn out to have been a baby tiger instead. I ask the reader to understand future uses of 'paradigm' as short for 'supposedly paradigm'. 
'tidying up' language aims to secure uniformity of use. To the extent to which it is successful in a language group, there is a correct use of language, and it is an objective matter to what one is and to what one is not committed by some sentence.

Words and sentence forms may be ambiguous, and new words and sentence forms enter language; but I shall count the language as having a correct use, so long as speakers can be got to recognise the ambiguity or novel meaning. This can often be achieved by philosophical discussion forcing a speaker to admit that in one sense of a word ' $W$ ', ' $S$ is $W$ ' is true, whereas in another sense ' $\mathrm{S}$ is $\mathrm{W}$ ' is false. Because of a lack of sensory or cognitive apparatus, some speakers do not have the capacity to extrapolate from any paradigm examples or inferential rules to the applicability of sentences in new situations. Some people are colourblind, and so unable to understand the sense of 'green' to which they have been introduced by examples of green objects and so apply it to new instances. Other people do not have the cognitive apparatus to recognize some philosophical or mathematical concept such as 'tensor product' or 'internal negation', to which they have been introduced, and so apply it to new instances. But so long as those who purport to be able to extrapolate from paradigm examples can be got to agree how to do so, I shall count the expressions as having an objective meaning in the language. I shall call the assumption that all sentences of the language would have, in consequence of these procedures, an objective meaning, the 'common language assumption'. I shall call a rule for what one is objectively committed to by a sentence, a rule of mini-entailment. S1 mini-entails s2 if and only if anyone who asserts s1 is thereby (in virtue of the rules for the correct use of language) committed to s2. S1 entails sn iff they can be joined by a chain of mini-entailments, such that s1 mini-entails some s2, s2 mini-entails some s3 and so on until we reach a sentence which minientails sn. I shall call a rule for what one is objectively not-committed to by a sentence or its negation a compatibility rule. $S 1$ is compatible with s2 iff s1 does not entail not-s2. If a sentence s1 is compatible with s2, it is of course compatible with all the entailments of s2.

II.

Among metaphysical necessities etc. are ones discoverable a priori, that is discoverable by mere reflection on what is involved in the claim made by the sentence. I'll call these logical necessities etc. (They include both 
'logical' necessities etc. in a narrow sense, and 'conceptual' necessities etc.) The obvious examples by which we learn the meaning of 'logically impossible sentence' are self-contradictory sentences and ones which entail self-contradictions. A self-contradictory sentence claims both that something is so and also that it is not so, for example, "he is taller than $6 \mathrm{ft}$ and it is not the case that he is taller than $6 \mathrm{ft}$.' For such a sentence could only be true if that something was so, and the sentence asserts that it is not so. No sentence could be more obviously or more strongly impossible than such a sentence; and any sentence which entails a selfcontradiction is as strongly impossible as a self-contradiction. And the natural understanding which most of us get from these examples is that a logically impossible sentence just is one which entails a selfcontradiction; and so any logically necessary sentence is one whose negation entails a self-contradiction, and any logically possible sentence is one which does not entail a self-contradiction.

Purported examples of logically necessary sentences whose negations do not entail a self-contradiction, turn out, I suggest, on examination, either to be such that their negations do entail a self-contradiction or not to be nearly as strongly necessary as ones whose negations entail a self-contradiction. ${ }^{2}$ And there is a general reason for denying that

${ }^{2}$ Robert Adams has one example of what, he writes, "seems to be a necessary truth": "Everything green has some spatial property". (See Robert Adams, 'Divine Necessity' republished in his The Virtue of Faith [Oxford: Oxford University Press, 1987], pp. 213-4.) He claims that this sentence cannot be shown to be 'analytic'. 'Analytic' may be understood in different ways, but one way which Adams mentions is being true "solely in virtue of the meanings of its terms"; and he claims that this account is "so vague as to be useless". But if 'analytic' is spelled out in terms of the negation of the sentence entailing a self-contradiction, the notion is clear. I suggest that being 'green' can be understood in two possible ways, and that the cited sentence with 'green' understood in either of these ways can be shown to be such that its negation entails a self-contradiction. Being green is a property of a thing. One can understand the word 'green' in such a way that a thing being 'green' entails that thing being a publicly visible thing. A publicly visible thing must have a spatial extension - for what one sees one sees as occupying a region of space. In that case the negation of the cited sentence clearly entails a self-contradiction. But one can understand 'green' in a sense in which (not merely a public visible thing, but also) a private thing experienced by only one person, the content of a mental event such as a sense-datum (or, less controversially, an after-image), could be 'green'. Clearly what it would be for that private thing to be green is to have the same visual appearance in respect of colour as a green public object. It must look like a surface or a volume which is green; and so must have the visual appearance of a spatial thing. For a private object to have a visual appearance of a spatial thing entails it looking as if it occupies a region of public space, and it can only do that if it occupies a spatial region of one's 
there are any logically impossible sentences other than ones which entail a self-contradiction (that is, any sentences which are as strongly impossible as those which entail a self-contradiction, and whose impossibility is detectable a priori, but which do not themselves entail a self-contradiction). The reason is that any such sentence must have the form of a declarative sentence, in which the component words already have a sense in the language. It will be a subject-predicate sentence, an existential generalization, or some other one of many recognized forms of declarative sentence. It will - to put the point loosely - assert something about some substance or property or event or whatever that it has or does not have some property or relation to some other substance, property, etc.; or that there are or are not certain substances, properties or whatever. Words have a sense in so far as it is clear what are the criteria for an object, property or whatever to be that object, property or whatever - they therefore delimit a boundary to the sort of object or property it can be or the sort of properties it can have. Hence it will be inconsistent to affirm that an object picked out by some expression is of a kind ruled out by the very criteria for being that object. And the form of a sentence s1 will exclude some alternative s2; and so it will be inconsistent to affirm (s1 \& s2). It follows therefore that sentences exemplifying what used to be called 'category mistakes', e.g. 'Caesar is a prime number' or 'this memory is violet' 3 are - in my sense - logically impossible sentences. If a sentence is not impossible for these reasons, then it will be making a claim about the world which does not entail a self-contradiction, a coherent claim. And plausibly no coherent claim can be as strongly impossible as a self-contradictory claim.

Given the common language assumption, we should all be able to agree - within a finite time - about many sentences that they entail selfcontradictions, and so are logically impossible; and about many sentences that they are such that their negations entail self-contradictions, and so are logically necessary. Compatibility rules also allow us to recognize many logically possible sentences; and so, since any sentence entailed

\footnotetext{
visual field. So again, even if one allows the existence of private objects which are green, the negation of 'everything green has some spatial property' entails a self-contradiction. Adams's example does not disconfirm my claim that the logically necessary is simply that the negation of which entails a self-contradiction, and that similar equivalences hold for logical possibility and impossibility.

${ }^{3}$ I take these examples from the article on 'category mistake' by Jack Meiland in R. Audi (ed.), The Cambridge Dictionary of Philosophy, Second Edition (Cambridge: Cambridge University Press, 1999), p. 123.
} 
by a logically possible sentence is itself logically possible, to recognize many more logically possible sentences. Of course a philosophical discussion often begins with disagreement about what entails what or what is compatible with what. The way to resolve a disagreement about whether $p$ entails $q$ is to find a route of mini-entailments from $p$ to $q$, or - alternatively - a route of mini-entailments from ( $p \&$ not- $q)$ to a self-contradiction. The way to resolve disagreements about whether $p$ is logically possible is to find some sentence (normally some long conjunction describing a circumstance) which disputants agree to be logically possible, which entails $p$ ), or to find some self-contradiction entailed by $p$. But prolonged failure to resolve disagreements in these ways is evidence of a failure in the common language assumption. That failure would mean that the examples and rules by which a word or sentence form has been given a sense has led to different unshareable concepts, different incommunicable understandings of that word or sentence form by different disputants. This may happen either because the two groups differ in their sensory life or in their cognitive abilities.

Here is one example. Suppose that the only noises humans could hear were noises produced by strings vibrating with different frequencies; and they then describe the noises produced by the more frequent vibrations as having a higher 'pitch'. The two groups might have two very different concepts of higher 'pitch'. One group's concept of a higher pitch might be simply the concept of being caused by a string vibrating more frequently; 'higher pitch' means more vibrations. The other group's concept might be that of a quality of a noise contingently caused by string vibration. Both groups would allow that everyone normally judges correctly which strings are vibrating more frequently, but that sometimes members of both groups make mistakes. Yet the two groups would describe the 'mistakes' differently. For the first group the mistakes are simply (bare) mistakes about how frequently the strings are vibrating, while for the second group the mistakes are mistakes caused by vibrations occasionally causing notes of different pitch from the ones they normally cause. For the first group the 'deaf' lack an ability to discriminate vibrations by means of their ears; for the second group the 'deaf' lack an ability to have auditory sensations. The different concepts of 'pitch' have different entailments. For the first group 'the string is now vibrating more frequently' entails 'it is producing a higher note': for the second group, it does not. And so on. The first group reports that it 'cannot make sense of' much of what the second group is claiming. 
The difference in the concepts inculcated by the teaching process may have arisen because the second group has sensations which the first group doesn't have. (The first group suffer from the auditory equivalent of blindsight.) But it may have arisen from a cognitive failure on the part of one or other group. It may be that both groups have sensations, but the first group doesn't have the ability to distinguish its sensations from its beliefs. Or it may be that neither group has sensations, but the second group suppose that they must be having sensations because they convince themselves from the example of vision that all perceptual beliefs must be mediated by sensations.

A different kind of example shows how lack of cognitive abilities alone may lead to different concepts. We are all taught by the same kinds of example what is a 'straight line'. Some people come to understand thereby simply a line (which can be extended indefinitely) such that the shortest distance between any two points on the line lies on the line, however far the line is extended. But others, while allowing that as a possible meaning for 'straight line' may acquire a more sophisticated understanding, that a 'straight line' is a line (which can be extended indefinitely) such that for any point $\mathrm{P}$ on the line there is some point $\mathrm{Q}$ on the line such that the shortest distance between $\mathrm{P}$ and any point on the line closer to $\mathrm{P}$ than $\mathrm{Q}$ lies on the line. This second understanding allows for the possibility of all straight lines (in this second sense) eventually returning to their starting point, and so there being no straight lines in the first sense, and so of space being unbounded but closed. Some people simply 'cannot make sense' of this possibility; for them being a 'straight line' in the second sense entails being a 'straight line' in the first sense. Other people can make sense of this possibility, and so deny the entailment. And, although I myself can make sense of it, it is always (epistemically) possible that I am deceived.

The only way to attempt to overcome such conflicts is to continue to pursue the methods described earlier - try harder to agree on logically possible sentences which entail a disputed sentence, or to find a route by which it leads to a self-contradiction. But it may be that some of us simply lack the ability to recognize certain modal truths, or - alternatively deceive ourselves into supposing that certain sentences are logically possible, when they are not. But where this doesn't happen, there will be agreement about what is logically impossible etc., so long as we have the same understanding of the 'logically impossible' as that which entails a self-contradiction. 
III.

But not all metaphysical impossibilities or necessities are logical impossibilities or necessities. In the 1970's, Kripke and Putnam ${ }^{4}$ drew our attention to the fact that there are many sentences which are such that neither they nor their negations seem to entail any self-contradiction, but which seem to be necessarily true or necessarily false with a necessity as strong as that oflogical necessity, but whose truth or falsity are discoverable only a posteriori. These sentences were said to be metaphysically but not logically, necessary or impossible. Thus, to modify an example used by Kripke to illustrate this class of sentences, suppose that in days long before people knew the geography of the Himalayas, explorers named a mountain of a certain visual appearance seen from Tibet 'Everest', and a mountain of a certain different shape seen from Nepal 'Gaurisanker', and used these names as rigid designators of the mountains. (A 'rigid designator' is a word which picks out the same object, however the object may change in respect of its non-essential properties.) These mountains are the same mountain; and being the mountains they are, they are - by the necessity of identity - necessarily the same mountain; and so - it seems - 'Everest is Gaurisanker' is necessarily true, with as hard as necessity as any logically necessary sentence. However - we may suppose - the explorers did not know this, and clearly would not have been able to discover its truth by mere a priori means. Hence it is not a logically necessary truth. Or consider Putnam's example of 'water is $\mathrm{H}_{2} \mathrm{O}$ ', 'water' being understood - as Putnam supposes that it was in the early nineteenth century - as a rigid designator of the transparent drinkable liquid in our rivers and seas. What makes the stuff that stuff is its chemical essence - being $\mathrm{H}_{2} \mathrm{O}$. Having that essence, it could not not have that essence. So 'water is $\mathrm{H}_{2} \mathrm{O}$ ' is metaphysically necessary, but again not so discoverable a priori. Hence is must be an a posteriori metaphysical necessity.

What has made these necessary sentences a posteriori is that the sentence contains at least one rigid designator of which we learn the meaning by being told that it applies to certain particular things

${ }^{4}$ Saul Kripke, Naming and Necessity, republished as a book (Oxford: Blackwell, 1980), and H. Putnam, 'The Meaning of "meaning", republished in his Mind, Language and Reality, Philosophical Papers, vol. 2 (Cambridge: Cambridge University Press, 1975). I interpret the claims of Kripke and Putnam about necessity etc. as claims about the necessity of sentences. Kripke makes it clear that his concern is with sentences, and writes that he has no 'official doctrine' of how his account applies to 'propositions'. (op. cit. pp. 20-21.) 
(especially substances and kinds of substances) having certain superficial properties, but where - we are told - what makes a thing that thing (that substance or a substance of that kind) is the essence (of which we may be ignorant) underlying those properties. In ignorance of the latter, we do not fully understand what we are saying about a substance when we say that it is that substance or a substance of that kind. Hence I shall call such designators 'uninformative designators'.

I define a rigid designator of a thing as an 'informative designator' if and only if someone who knows what the designator means (that is, has the linguistic knowledge of how to use it) knows a certain set of conditions necessary and sufficient (in any logically possible world) for a thing to be that thing (whether or not he can state those conditions in words.) I define a rigid designator as an 'uninformative designator' if and only if these conditions are not satisfied. To 'know' these conditions for the application of a designator - as I shall understand this expression just is to be able (when favourably positioned, with faculties in working order, and not subject to illusion) to recognize where the informative designator (or, if it is defined in words, the words by which it is defined) applies and where it does not and to know the mini-entailments of sentences in which it occurs. Having the ability to recognize something when favourably positioned with faculties in working order and not subject to illusion, involves knowing what that thing is. In the case of technical terms, it is experts in the relevant field whose knowledge of the relevant necessary and sufficient conditions determines the meaning of a term. Thus it is physical scientists, whose knowledge determines the meanings of 'quark' or 'electron'.

Many of the words - for example 'red,' 'square,' 'has a length of 1 metre' - by which we pick out properties are informative designators; they are such that if we know what the words mean we can recognize (subject to the stated restriction) where they do or do not apply, and can make the requisite inferences. Other words by which we pick out properties can be defined by words for which those conditions hold. For example 'has a length of $10^{-15}$ metres' can be defined in terms of the informatively designated property 'has a length of 10 metres' and the informatively designated relation of 'being shorter by $1 / 10^{\text {th }}$ than' (used 15 times).

So the reason why the claims originally made by the sentences 'Everest is Gaurisanker' and 'water is $\mathrm{H}_{2} \mathrm{O}$ ' are necessary with as hard a necessity as 'logical necessity' is that they are logically necessary, but the use of uninformative designators has the consequences that speakers 
did not know fully what these claims were until they had done some a posteriori investigation. When we know fully what we are talking about, mere a priori considerations can show whether some sentence is metaphysically necessary or impossible. Hence there is available a definition of a sentence as metaphysically necessary (impossible or possible) iff it is logically necessary (impossible or possible) when we substitute co-referring informative designators for uninformative designators. This definition will capture as metaphysically necessary (impossible or possible) almost all the examples of the 'metaphysically necessary' ('impossible' or 'possible') offered by Kripke, Putnam, and others. And so from these examples we derive a sense of metaphysically necessary in which a sentence is metaphysically necessary (impossible or possible) iff it is logically necessary (impossible or possible) when informative designators are substituted for uninformative designators. And so, given the earlier understanding of 'logical impossibility', and the understanding of metaphysical impossibility as in reality logical impossibility, it would seem that no sentence could be as strongly impossible metaphysically as one which is in reality logically impossible; and so there can be no metaphysically impossible (necessary, or possible) sentences apart from ones of the kind analysed in this section.

IV.

So, given some a posteriori logically contingent information (e.g. about which are the molecules of which whatever is the transparent stuff in our rivers and seas is made) which determine which sentences are metaphysically necessary, there should be no scope for disagreement about the modal metaphysical status of any sentence - given that the common language assumption applies to the words and sentence forms of the language - and that we have the same understandings of 'logical' and 'metaphysical' impossibility.

Now I have introduced the term 'metaphysically' impossible as the strongest kind of impossibility which a sentence can have; and defined the 'logically' impossible in terms of the metaphysically impossible. I have filled out what it is for a sentence to be impossible in this 'strongest' sense by examples of self-contradictions, entailments, and compatibilities; and by means of Kripke - Putnam type examples, which I have described in my own way by means of the concept of an 'informative designator'. The particular examples could form the basis of any philosophy student's 
introduction to the concepts of metaphysical and logical impossibility. From these examples I have derived sharp usable senses of 'logically impossible' ('entailing a contradiction') and 'metaphysically impossible' (reducible in the stated way to 'logically impossible'), and thereby of the other modal concepts. I call these senses of the terms 'logically' and 'metaphysically' the narrow senses, and I will assume them for the rest of this section.

Others may purport to derive from the paradigm examples wider understandings of these terms. They may suppose (for example) that there are impossibilities in the strongest sense detectable a priori which do not entail contradictions; or necessities in the strongest sense which do not reduce to sentences whose negations entail contradictions when we substitute informative for uninformative designators. ${ }^{5}$ The issue then arises whether it is logically possible (on my understanding of this concept) for there to be such impossibilities or necessities. And the only way to resolve any disagreement about this is by the methods described earlier: putting forward examples which we can both recognize do not entail self-contradictions, or showing by a route that we can both recognize that purported examples do entail self-contradictions. But I am pessimistic about the chances of my reaching agreement with many of my opponents on this matter within any finite time. That will show that one or other of us suffers from some (sensory, or much more likely) cognitive deficiency.

\section{V.}

However, given my understanding of these concepts together with my assumption that the modal properties are properties of sentences, it seems fairly implausible to suppose that a (positive) existential sentence (a sentence claiming that there exists some thing or things of a certain

\footnotetext{
${ }^{5}$ Gendler and Hawthorne write, that "the notion of metaphysical possibility ... is standardly taken to be primitive", adding in a footnote "in contemporary discussions at any rate" ([eds] T. Gendler and J. Hawthorne, Conceivability and Possibility [Oxford: Oxford University Press, 2002], Introduction, p. 4.). It doesn't help me to understand this notion for them to say that it is the most basic conception of 'how things might have been' (ibid. pp. 4-5.). For since this 'most basic conception' is supposed to be wider than logical possibility (as defined by me), it is unclear how the latter is to be widened unless in the way I have analysed. (In one book I myself unhelpfully used 'metaphysically necessary' to mean [roughly] whatever is the ultimate cause of things or is entailed by the existence of that ultimate cause; and so the 'metaphysically possible' is whatever is compatible with the existence of the actual ultimate cause. See The Christian God [Oxford: Oxford University Press, 1994], pp. 118-19. But this is certainly not the sense which most writers who use the term have in mind, and not the sense in which I am using in this paper.)
} 
kind) can be logically necessary - for to be so it would need to be such that its negation entails a self-contradiction. The negation of an existential sentence has the form ' $\sim(\exists \mathrm{x})(\phi \mathrm{x})$ '; it claims that a certain property (or conjunction of properties) is not instantiated. A self-contradictory sentence, of a kind not containing modal operators, claims that the actual world has a contradictory quality, and that will be so either because some object within it has such a quality or because it both does and does not contain an object of a certain kind. So it will have the form or entail a sentence of the form ' $(\exists \mathrm{x})(\psi \mathrm{x}) \& \sim(\exists \mathrm{x})(\psi \mathrm{x})$ ' or the form ' $(\exists \mathrm{x})(\psi \mathrm{x} \&$ $\sim \psi \mathrm{x})$ '. Either way it will include or entail a positive existential sentence. But plausibly the mere non-existence of anything of some kind cannot entail the existence of anything. It may be suggested that the contradiction has the form of a sentence in which there are modal operators, where the contradiction arises from its modal features; for example a sentence of the form 'it is possible that something is $\phi$, and it is not possible that something is $\phi, ' \diamond(\exists \mathrm{x})(\Phi \mathrm{x}) \& \sim \nabla(\exists \mathrm{x})(\Phi \mathrm{x})$ '. But it is not easy to see how the mere non-existence of anything of some kind could entail a modal sentence which would not be entailed by the existence of a thing of that kind.

The plausible suggestion that the mere non-existence of anything of some kind cannot entail a contradiction, and so no positive existential sentence can be a necessary truth is of course is of course due to Hume. ${ }^{6}$ It will hold whether the thing is of a concrete or an abstract kind. So the supposed necessary existential truths of arithmetic do not constitute an exception. The negation of, for example, such a supposed necessary truth as 'There are prime numbers greater than 3', 'There are no prime numbers greater than 3', does not by itself entail a contradiction; it does so only when conjoined with some existential axiom of arithmetic (e.g 'There is a number 1' and 'Every number has a successor'). So my suggestion must hold also for the special case where ' $\varnothing$ ' designates any conjunction of properties of a kind supposed to constitute a definite description of God - e.g. 'omnipotent, omniscient, perfectly good, and eternal' - and so God cannot be a logically necessary being.

However some people claim (in effect) that a particular negative existential sentence of this kind does entail a contradiction, and some of them claim to have demonstrated this. If this disagreement persists after serious attempts to clarify the issues, this indicates another case

6 "There is no being ... whose non-existence implies a contradiction." D. Hume, Dialogues Concerning Natural Religion, originally published 1779 (New York, Hafner Publishing Co., 1948), Part 9, p. 58. 
where my 'common language assumption' is mistaken. The entailment must depend on understanding sentences in different senses from the way the rest of us understand them, senses which are not equivalent to any which we can grasp. Yet if someone claims that 'there is no $\mathrm{x}$ such that $\varnothing \mathrm{x}$ ' (where ' $\varnothing$ ' designates a definite description of God of any traditional kind) entails a contradiction, they will need to hold that innumerable other sentences of very different kinds to each other entail contradictions, when the rest of us hold that they are obviously logically possible. For example they will need to hold that 'Once upon a time there were no rational beings', and 'No one knows everything', and 'No one is perfectly good', and - among sentences evidently describing nonactual worlds - 'The only substances are four mutually repelling steel balls', and 'No one knows what is happening outside a sphere of 1 mile diameter surrounding their body' all entail contradictions, because - if any one of them does not entail a contradiction - there would not be a God in anything like a traditional sense. Someone could only derive a contradiction from all such sentences if they understood an enormous number of predicates - not just one or two technical philosophical terms - in different senses from the rest of us, or if they understood one or more formal terms such as 'exists', 'not', or 'all' differently from the rest of us. In view of the similarity in respect of psychological make-up and the process of language acquisition between humans who believe in ontological arguments and those who don't, I do not find it very plausible to suppose that the former understand all these predicates in different senses from the rest of us. But if they do understand the predicates in the same senses as the rest of us, they will have to admit that 'there is no God' does not entail a self-contradiction, and so 'there is a God' cannot be logically necessary in the sense in which I have spelled it out.

But if there is no logically necessary sentence of the form 'there is an $\mathrm{x}$ such that $\varnothing \mathrm{x}$ ' where ' $\varnothing$ ' designates a definite description of God, no substitution for 'God' in 'God exists' of a co-referring designator will yield a logically necessary sentence, and so 'God exists' cannot be metaphysically necessary in the sense in which I have spelled it out.

VI.

My arguments so far assume that the primary bearers of modal properties are sentences of human languages. Because many human languages have very similar structures to each other - words introduced by the same observable circumstances, the same types of sentences (subject-predicate 
sentences, existential sentences, and so on) with parallel inference patterns between them, some sentence in one language often means the same as some sentence in another language; they are inter-translatable. And so in order to talk about the claim which would be made by any such sentence, or any other sentence meaning the same which might be uttered in a language not yet invented, it is useful to suppose that - even before such a claim is made - there is a common thing which they all express, a proposition, the content and logical consequences of which can be discussed independently of the particular language in which it is expressed. This however is merely a useful fiction. There is no reason to suppose that there really are such things as propositions, existing independently of the sentences which express them. We cannot interact with propositions, nor do we need to postulate them in order to explain what we observe - the behaviour of humans who utter sentences. And if the necessary truths were truths about eternal propositions, there would be no easy explanation of how we are in a position to know which such propositions are necessarily true. Why should we trust our intuitions about this Platonic realm? Whereas if necessary truths are truths about human language, there is a ready explanation of how we are in a position to know about them: we learn them in learning language. So there is no reason to deny that ordinary talk about 'propositions' (of a kind that does not imply their eternal existence) can be analysed as talk about human sentences.

If however we suppose that propositions are real timeless entities which have a modal status independently of any human sentences which might express them, then there is some plausibility in the claim that the proposition expressed (imperfectly) by the sentence 'there is a God' might be a metaphysically necessary truth - even if we assume that logically impossible propositions are ones which entail a self-contradiction, and metaphysically impossible propositions are ones from which we can derive from logically impossible propositions by substituting co-referring informative for uninformative designators. For there will not be the slightest reason to suppose that there are only as many propositions as will eventually be expressed or even (in some sense) could be expressed. In that case there would not be any reason to suppose that all necessary propositions which can be expressed can be shown by us to be necessary, because the demonstrations thereof may depend on a deduction which proceeds by means of propositions of kinds which cannot be expressed and whose mini-entailments may be known only to superior beings. 
So maybe 'God exists' is necessarily true - even though we humans are totally unable to show that. That of course would not provide us with a sound ontological argument, but it does allow the possibility of there being one, unknowable by humans.

This way of thinking does however carry certain unwelcome consequences for theism. It looks as if all these other necessary propositions coexisting eternally with God, constrain how God can act. If for example it is a necessarily true proposition that God cannot make me exist or not exist at the same time, then this constrains what God can do - not merely what we can do with the English language without uttering a necessarily false sentence. That necessarily true proposition would limit God. One way of attempting to avoid this is to claim that necessarily these propositions are ideas in the mind of God, and so part of his nature. But that raises the question why these propositions rather than any other ones are part of God's nature, and if the answer is that it is just a brute fact that they are, that would make God essentially a very un-simple being. Alternatively one could suppose that God has a nature such that necessarily he creates just these necessary truths. ${ }^{7}$ On a normal theistic view God is necessarily good, and so the only necessary truths which it follows from his essence that he will create will be good ones. But there are well-known difficulties in supposing that any agent (including God) can make actions (morally) good (except in virtue of some fundamental moral principle which lays down which actions an agent can make good under which circumstances - e.g. that it is good to use force to uphold a just law if commanded to do so by a just sovereign). ${ }^{8}$ Given that the fundamental principles about

${ }^{7}$ See for example Thomas Morris and Christopher Menzel 'Absolute Creation' in (ed.) T. Morris, Anselmian Explorations (Notre Dame, Indiana: University of Notre Dame Press, 1987), pp. 161-172. The later pages of this paper (pp. 172-8) go on to defend a (to my mind) even more implausible correlate of this view - that God creates his own nature.

${ }^{8}$ For example, it does rather look as if some of the same actions would be good or bad if there was no God, as are good and bad if there is a God. But if no action would be good or bad unless God had so willed it, that must be because God has some property which other persons lack (e.g. being our creator, or being omnipotent) so that God's willing some action would make this difference whereas other persons willing it would not. But then there must be a fundamental principle independent of God, that it is good to do any action willed by someone having that property; and that principle couldn't be true in virtue of being willed by God. For my account of the relation of God to morality see my 'What difference does God make to morality?' in (ed.) R.K. Garcia, Is Goodness without God good enough? (Lanham, Maryland: Rowman and Littlefield, 2009), pp. 151-63; and my Responsibility and Atonement (Oxford: Oxford University Press, 1989), chapters 1 and 8. 
which actions are good (that is, would be good if they were performed) are independent of the actions of agents, the fundamental principles to which propositions it is good to award the status of necessary truth will be independent of the actions of God. On the propositional view these will be eternally necessary propositions (stating which propositions it would be a good action to make necessary) existing independently of the actions of God, and determining which actions he can do. But then there will be necessarily true eternal propositions independent of the will of God stating which actions (of creating necessary truths) these are. These propositions determine how God can act; they cannot be consequences of God's actions. But if necessary truths (including the fundamental moral truths) are just truths of human language about human language (including the truth about which property 'morally good' designates, and so which properties, such as the property of feeding the starving, are entailed by it), there are no pre-existing things apart from God - although of course the states of affairs which human language is used to describe - e.g. that God is good - may exist before they are described by humans.

So for all these reasons we should regard logical necessity as belonging primarily to human sentences, and only to any other entities as a convenient fiction; and then, I suggest, it follows that God is not a metaphysically necessary being (in the sense analysed in this paper), because it is not logically possible (in my sense) that there be any metaphysically necessary being. ${ }^{9}$ But this fact has no relevance to the

${ }^{9}$ I very much doubt whether anyone earlier than Anselm thought that God is a metaphysically necessary being in the sense being discussed. Aquinas did not use 'necessary being' in this sense. For in his Summa Theologiae Ia. 50.5 ad. 3 (as elsewhere) he clearly implies that, as well as God, angels (which are beings created by the voluntary act of God) are also necessary beings. He seems to think of a necessary being as one not subject to corruption, that is, one which will go on existing forever unless caused not to exist by something else. He distinguished God from other necessary beings as a "being necessary through its own nature (per se) and not caused to be necessary by something else" (op. cit. Ia. 2.3) and so 'unconditionally necessary'. Angels depend for their noncorruptibility on God and so are only 'conditionally necessary'. However Aquinas also seems to claim (in effect) that only self-contradictions are absolutely impossible. ("The impossible is that in which the predicate is incompatible with the subject" - op.cit. Ia. 25.3.) That might seem to suggest that he thought that the negation of 'there is a God' entailed a contradiction, and so he did - but that was because he thought that anything incompatible with what was already fixed entailed a contradiction; on his view what is absolutely possible changes with time. But God, as the eternal source of everything, is always fixed, and so - by Aquinas's criteria - his non-existence is always impossible, and that is why he is absolutely necessary. (I am indebted for this analysis of Aquinas's 
logical possibility of there existing a being necessary in some other sense, e.g. a being essentially everlasting and essentially not causally contingent on the existence of any other being for its own existence, which is a property which all traditional theists have believed God to have. And this fact allows the possibility of there being a cogent inductive argument to the existence of such a being. ${ }^{10}$

understanding of modal concepts to Brian Leftow. See his paper Aquinas, Divine Simplicity, and Divine Freedom' in [ed.] K. Timpe, Metaphysics and God [London: Routledge, 2009], esp. pp. 23-29.) But this is not metaphysical necessity in the sense in which I have been discussing it, which derives from a sense of logical necessity in which 'entailing a contradiction' is something intrinsic to a sentence (with its meaning), and independent of what is or is not already fixed outside the sentence. Admittedly, Aquinas also thought that "God is the same as his own nature or essence" (Ia.3.3); but he goes on to claim that anything immaterial, not just God, is the same as its own nature. His point is simply that material things are individuated by the matter of which they are made, whereas immaterial things are individuated by their forms, that is natures. I know of nothing in Aquinas which should lead us to suppose that he thought that God's existence is a metaphysically necessary truth (in the sense used in this paper). He certainly thought that on Anselm's 'definition' of God the negation of 'There is a God' did not entail a selfcontradiction (Ia. 2.1. ad. 2), and I know of no reason to suppose that he thought that this would hold on any other 'definition' (in our sense) of 'God'.

${ }^{10}$ For my own inductive arguments to the existence of God of this kind, see my The Existence of God, Second Edition (Oxford: Oxford University Press, 2004). 